\title{
Teleportation of a Bose-Einstein condensate state by controlled elastic collisions
}

\author{
M. C. de Oliveira \\ Departamento de Física, CCET, Universidade Federal de São Carlos, \\ Via Washington Luiz km 235, 13565-905, São Carlos, SP, Brazil.
}

(December 4, 2018)

\begin{abstract}
A protocol for teleportation of the state of a Bose-Einstein condensate trapped in a three-well potential is developed. The protocol uses hard-sphere cross-collision between the condensate modes as a means of generating entanglement. As Bell state measurement, it is proposed that a homodyne detection of the condensate quadrature is performed through Josephson coupling of the condensate mode to another mode in a neighbouring well.
\end{abstract}

03.67.Hk, 03.75.Fi, 32.80.Pj

\section{INTRODUCTION}

Teleportation of quantum states, proposed by Bennett et al. [1], was first realised for light polarization states [2], owing to the possibility of generating nonlocal entanglement between parties (the quantum channel) in this system [3]. Although many proposals and experimental realizations of nonlocal entanglement of massive particles (atoms and ions) exist [4], up to now there has been no experimental evidence of the teleportation of massive particles state [5]. Indeed, non-linear interactions (a valuable resource for deterministic generation of entanglement) are always present in many-particle systems. An interesting question then arises - to what extent can the teleportation protocol be applied to massive many-particle systems? A strong candidate for massive particle state teleportation is the condensate state of a matter field, where non-linear interactions appear quite naturally as elastic collisions [6].

In this paper we propose an experimental protocol for teleportation [1] of mode states of an atomic BoseEinstein condensate (BEC) trapped in an optical lattice potential, by using controllable elastic collisions and Josephson coupling [7] between modes for both the quantum channel formation and measurement stage. Elastic collisions are a fundamental resource for both the formation of the entanglement and the parity operations needed to correct the teleported state. For the measurement stage, we propose a balanced homodyne detection of the BEC modes quadratures, valid for a small condensate. By measuring the difference of population in two condensate modes (the central mode and a reference mode) interacting via Josephson coupling the central mode quadrature is determined.

This paper is organized as follows. In Sec. II we present the model for three interacting condensate modes trapped in a three-well potential. In Sec. III we propose the teleportation protocol using controlled collisions as a means to generate entanglement. In Sec. IV we turn to the measurement stage, presenting a scheme of homodyne detection of the BEC phase. In Sec. V we present the operations of parity and displacement needed in order to correct to the required state, the state of the condensate mode at the receiver station. In Sec. VI we present a physical implementation of the controlled collision on optical lattices. In Sec. VII a discussion encloses the paper.

\section{MODEL}

The observation of BECs of diluted trapped neutral atoms [8] and the recent achievement of all-optical confinement of a BEC [9] and condensation on a microelectronic chip [10] have stimulated a large research program on BEC of diluted neutral atom gases. Of particular interest is the study of a BEC in a confining potential. In [11] the dynamics of a BEC in a double-well potential was modelled. In that model, coherent oscillations due to tunnelling (Josephson-like coupling) [7 between the two wells are suppressed when the number of atoms exceeds a critical value (self-trapping). In fact, the barrier separation between the two wells has a central role as it determines whether the Josephson coupling between modes is negligible in contrast to cross-collisions, when the wave-functions of the two modes considerably overlap. A dynamic process can be envisaged where the twowell barrier is lowered and raised back adiabatically, such that the elastic collisions leave the two modes in an entangled state [12] - a hallmark of teleportation protocols. This is the mechanism we focus on here. We extensively use controlled collisions between condensate modes, by adiabatically turning off and on the potential barriers in an optical lattice potential, in order to teleport the state of one condensate mode, to another mode, located inside the trap, but delocalised from the first one. This sequential process is depicted in Fig. 1. Initially we describe the general approach for entanglement generation and measurement and at the end we give a specific but clarifying physical implementation of the time varying potential, for optical lattices. 


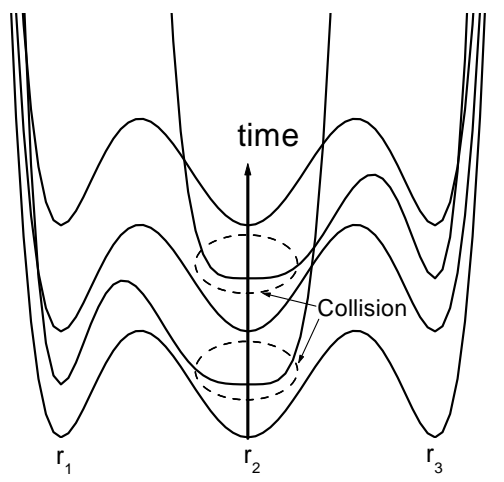

Fig.1. Sequential collision for entanglement formation.

Extending the model in [11, we consider a BEC trapped in a symmetric three-well single-particle potential $V(r)$ with minima at $r_{1}, r_{2}$ and $r_{3}$ disposed along the $z$ axis. We assume that the three lowest states of the potential are closely spaced and well separated from its higher levels, and that many-particle interactions do not significantly change this situation, to allow a threemode approximation. The potential expanded around each minimum is

$$
V(\mathbf{r})=\widetilde{V}^{(2)}\left(\mathbf{r}-\mathbf{r}_{j}\right)+\ldots j=1,2,3,
$$

where $\widetilde{V}^{(2)}\left(\mathbf{r}-\mathbf{r}_{j}\right)$ is the parabolic approximation to the potential in the vicinity of each minimum. The normalized single-particle ground-state $u_{0}(\mathbf{r})$ of the local potential $\widetilde{V}^{2}(\mathbf{r})$, with energy $E_{0}$, defines the local mode solutions of the individual wells. If the position uncertainty in the state $u_{0}(\mathbf{r})$ is much less than the separation of the minima of the global potential, the overlap between the modes of each well, $\epsilon$, is much less than unity and the modes are approximately orthogonal [11]. The many-body Hamiltonian describing an atomic BEC in this potential is

$$
\begin{aligned}
H= & \int d^{3} x \psi^{\dagger}(\mathbf{r})\left(-\frac{\hbar}{2 m} \nabla^{2}+V(\mathbf{r})\right) \psi(\mathbf{r}) \\
& +\frac{1}{2} \frac{4 \pi a_{s} \hbar^{2}}{m} \int d^{3} r \psi^{\dagger}(\mathbf{r}) \psi^{\dagger}(\mathbf{r}) \psi(\mathbf{r}) \psi(\mathbf{r}),
\end{aligned}
$$

where $m$ is the atomic mass, $U_{0}=4 \pi \hbar^{2} a / m$ measures the strength of the two-body interaction, $a$ being the s-wave scattering length, $\psi(\mathbf{r}, t)$ and $\psi^{\dagger}(\mathbf{r}, t)$ are the Heisenberg picture field operators, which annihilate and create atoms at position r, and normal ordering has been used. In the three-mode approximation the field operators are expanded in terms of the local modes and the Heisenberg picture annihilation and creation operators read as

$$
c_{j}(t)=\int d^{3} \mathbf{r} u_{j}^{*}(\mathbf{r}) \psi(\mathbf{r}, t)
$$

so that $\left[c_{j}, c_{k}\right]=\delta_{j k}$ to order $\epsilon^{0}$. With this prescription, and retaining terms up to order $\epsilon$, one arrives at two distinct regimes: (i) If the potential wells are well separated only self-collision terms are important and the many-body Hamiltonian reduces to

$$
\begin{aligned}
H_{1}= & E_{0}\left(c_{1}^{\dagger} c_{1}+c_{2}^{\dagger} c_{2}+c_{3}^{\dagger} c_{3}\right) \\
& +\hbar \kappa\left[\left(c_{1}^{\dagger}\right)^{2} c_{1}^{2}+\left(c_{2}^{\dagger}\right)^{2} c_{2}^{2}+\left(c_{3}^{\dagger}\right)^{2} c_{3}^{2}\right],
\end{aligned}
$$

where $\kappa=U_{0} / 2 \hbar V_{e f f}$, and $V_{e f f}^{-1}=\int d^{3} \mathbf{r}\left|u_{0}(\mathbf{r})\right|^{4}$ is the effective mode volume of each well. In such a situation, no cross-collisions or Josephson tunnelling occur. (ii) If the potential wells are not well separated Josephson tunnelling [7] between neighbour wells occurs and in the diluted atomic gas regime it prevails over cross-collisions. The many-body Hamiltonian then becomes

$$
\begin{aligned}
H_{2}= & E_{0}\left(c_{1}^{\dagger} c_{1}+c_{2}^{\dagger} c_{2}+c_{3}^{\dagger} c_{3}\right)+\hbar \frac{\Omega}{2}\left(c_{1}^{\dagger} c_{2}+c_{2}^{\dagger} c_{3}+H . c .\right) \\
& +\hbar \kappa\left[\left(c_{1}^{\dagger}\right)^{2} c_{1}^{2}+\left(c_{2}^{\dagger}\right)^{2} c_{2}^{2}+\left(c_{3}^{\dagger}\right)^{2} c_{3}^{2}\right],
\end{aligned}
$$

where $\Omega=2 \mathcal{R} / \hbar$ is the tunnelling frequency between two minima, with

$$
\mathcal{R}=\int d^{3} \mathbf{r} u_{i}^{*}(\mathbf{r})\left[V(\mathbf{r})-\widetilde{V}^{(2)}\left(\mathbf{r}-\mathbf{r}_{i}\right)\right] u_{i+1}(\mathbf{r}), \quad i=1,2 .
$$

We assume that in equilibrium state, regime (i) rules out, and the modes can be treated independently. If the barrier separating modes 2 and 3 is lowered adiabatically $\left(\frac{d V}{d t} \ll\left(E^{\prime}-E_{0}\right) / \hbar\right)$, in order to avoid transitions to other states (of energy $E^{\prime}$ ), the two respective modes overlap and strong cross collision occurs as $V_{c}=2 \hbar \kappa c_{2}^{\dagger} c_{2} c_{3}^{\dagger} c_{3}$. When the barrier is raised back adiabatically, bringing the system to the equilibrium regime, the two modes split again, but now having a non-local entanglement generated by the cross collision term, over the time the two modes overlapped. In such a case a non-local quantum channel would be formed between condensate modes 2 and 3 [13] as we describe in next section.

\section{TELEPORTATION PROTOCOL}

The following protocol is more efficient if the condensate modes are initially prepared in coherent states, but we may assume a general initial state expanding it in the coherent state basis, $\left|\psi_{a}\right\rangle_{2}=\int d^{2} \alpha a_{\alpha}|\alpha\rangle_{2}$ and $\left|\psi_{b}\right\rangle_{3}=\int d^{2} \beta b_{\beta}|\beta\rangle_{3}$, for modes 2 and 3 respectively. The dynamics governed by $H_{1}$, together with the crosscollision $V_{c}$, gives for modes 2 and 3 alone

$$
\begin{aligned}
|\psi(t)\rangle= & \int d^{2} \alpha d^{2} \beta a_{\alpha} b_{\beta} e^{-\left(|\alpha|^{2}+|\beta|^{2}\right) / 2} \\
& \times \sum_{m, n=0}^{\infty} \frac{1}{\sqrt{m ! n !}}\left(\alpha e^{-i\left(E_{0} / \hbar-\kappa\right) t} e^{-i \kappa m t}\right)^{m}
\end{aligned}
$$




$$
\times\left(\beta e^{-i\left(E_{0} / \hbar-\kappa\right) t} e^{-i \kappa(m+n) t}\right)^{n}|m\rangle_{2}|n\rangle_{3},
$$

which, for $t=\pi / 2 \kappa$, turns out to be the entangled state given by

$$
\begin{aligned}
|\Phi(\pi / \kappa)\rangle= & \frac{1}{2} \int d^{2} \alpha d^{2} \beta a_{\alpha} b_{\beta}\left[(1-i)\left|\alpha e^{-i \phi}\right\rangle_{2}\left|\beta e^{-i \phi}\right\rangle_{3}\right. \\
& \left.+(1+i)\left|-\alpha e^{-i \phi}\right\rangle_{2}\left|-\beta e^{-i \phi}\right\rangle_{3}\right]
\end{aligned}
$$

where $\phi=\left(E_{0} / \hbar-\kappa\right) / 2 \kappa$. Choosing properly the frequency of the modes, $\left(E_{0} / \hbar-\kappa\right)$, a set of approximately orthonormal states $\left|\Phi_{j}\right\rangle$ can be generated for $E_{0} / \hbar=(j+1) \kappa, j=0,1,2,3$, respectively as

$$
\begin{aligned}
\left|\Phi_{j}\right\rangle= & \frac{1}{2} \int d^{2} \alpha d^{2} \beta a_{\alpha} b_{\beta}\left[(1-i)\left|(-i)^{j} \alpha\right\rangle_{2}\left|(-i)^{j} \beta\right\rangle_{3}\right. \\
& \left.+(1+i)\left|-(-i)^{j} \alpha\right\rangle_{2}\left|-(i)^{j} \beta\right\rangle_{3}\right] .
\end{aligned}
$$

From now on we suppose that the condition for the generation of $\left|\Phi_{0}\right\rangle$, i.e., $E_{0} / \hbar=\kappa$, is met.

A simple teleportation protocol [13] based on homodyne measurement of the condensate modes phases can be performed. Let us say the condensate mode 1, called hereafter the target mode, is prepared in an unknown superposition of the specific form 14]

$$
|\psi\rangle_{T}=\int d^{2} \gamma c_{\gamma}(A|\gamma\rangle+B|-\gamma\rangle)
$$

where $A$ and $B$ are constants respecting normalization conditions. Now if the modes 1 and 2 are made to collide the whole condensate state is left as

$$
\begin{aligned}
& \frac{1}{2} \int d^{2} \alpha d^{2} \beta d^{\gamma} a_{\alpha} b_{\beta} c_{\gamma}\{-i|\gamma\rangle|\alpha\rangle(A|\beta\rangle-B|-\beta\rangle) \\
& +|\gamma\rangle|-\alpha\rangle(A|-\beta\rangle+B|\beta\rangle)+i|-\gamma\rangle|\alpha\rangle(A|-\beta\rangle-B|\beta\rangle) \\
& +|-\gamma\rangle|-\alpha\rangle(A|\beta\rangle+B|-\beta\rangle)\},
\end{aligned}
$$

a three-partite entangled state composed of four elements. We can distinguish each element by the phase of modes 2 and target. Notice that although the protocol is encoded in continuous variables states, the protocol itself is discrete as only four equiprobable outcomes are possible. Thus after the distinction of the target and mode 2 phases, only two bits of classical information have to be sent to the mode 3 at the receiver station.

\section{HOMODYNE DETECTION OF BEC PHASE}

If a joint measurement on both modes 1 and 2 can be envisaged to distinguish the phase of each condensate mode, the complete Bell state measurement is realised. Tomographic reconstruction of the condensate state would allow the distinction between the two different phases. Such an approach is based on optical homodyne tomography [15], using an arrangement composed of an atomic beam splitter and an ideal atom counter. Here instead, we describe an alternative scheme for phase determination similar the optical balanced homodyne measurement, where the Josephson coupling [7] plays the role of an atomic beam splitter. Consider two condensate modes separated by a barrier, as in 11]. A two-mode approximation is assumed. Neglecting cross-collision terms (once the overlap of condensate wave-functions is negligible) the Hamiltonian for the two modes is

$$
\begin{aligned}
H= & E_{0}\left(c^{\dagger} c+b^{\dagger} b\right)+\frac{\hbar \Omega}{2}\left(c^{\dagger} b+b^{\dagger} c\right) \\
& +\hbar \kappa\left[\left(c^{\dagger}\right)^{2} c^{2}+\left(b^{\dagger}\right)^{2} b^{2}\right],
\end{aligned}
$$

Defining new operators as $S_{x}=\frac{1}{2 N}\left(c^{\dagger} c-b^{\dagger} b\right), S_{y}=$ $\frac{i}{2 N}\left(c^{\dagger} b-c b^{\dagger}\right), S_{z}=\frac{1}{2 N}\left(c^{\dagger} b+c b^{\dagger}\right)$, where $N=\left\langle c^{\dagger} c+b^{\dagger} b\right\rangle$, the equations for the evolution of these operators are

$$
\begin{aligned}
& \dot{S}_{x}=-\Omega S_{y}, \\
& \dot{S}_{y}=\Omega S_{x}-2 i \epsilon \Omega S_{y}-4 \epsilon \Omega N S_{x} S_{z}, \\
& \dot{S}_{z}=-2 i \epsilon \Omega S_{z}+4 \epsilon \Omega N S_{x} S_{y},
\end{aligned}
$$

where $\epsilon=\frac{\kappa}{\Omega} \ll 1$. A semiclassical solution for $S_{x}$ is given up to first-order in $\epsilon$ by

$$
\begin{aligned}
S_{x}(t)= & {\left[S_{x}(0)+\epsilon t\left(2 N z_{0} y_{0}-i x_{0}\right)\right] \cos \Omega t } \\
& -\left[S_{y}(0)-\epsilon t\left(2 N z_{0} x_{0}+i y_{0}\right)\right] \sin \Omega t,
\end{aligned}
$$

valid only for $\epsilon N \ll 1$, i.e., for $\kappa \ll \Omega$, a small number of particles and also for a short time. To derive this solution the above operators are expanded as $S_{x}=\sum_{n} \epsilon_{n} x_{n}$, $S_{y}=\sum_{n} \epsilon_{n} y_{n}$ and $S_{z}=\sum_{n} \epsilon_{n} z_{n}$. Assuming initially an equal number of atoms in both wells, the solution (16) simplifies to

$$
S_{x}(t)=-S_{y}(0) \sin \Omega t+2 \epsilon t N z_{0} y_{0} \cos \Omega t+i \epsilon t y_{0} \sin \Omega t .
$$

Consider the following semiclassical picture for the operator $S_{y}$

$$
\left\langle S_{y}\right\rangle=\frac{i}{2}|\beta|\left(\left\langle c^{\dagger}\right\rangle e^{i \theta}-\langle c\rangle e^{-i \theta}\right)=-|\beta|\left\langle X_{\theta-\pi / 2}\right\rangle,
$$

where the mode $\mathrm{B}$ was prepared in a coherent state given by $\beta=|\beta| e^{i \theta}$. It is easy to observe then that for $\epsilon N \ll 1$, at $t=\pi / 2 \Omega$, the Eq.(17) gives the well-known result for balanced homodyne detection, plus a small correction

$$
\left\langle S_{x}(\pi / 2 \Omega)\right\rangle=|\beta|\left\langle X_{\theta-\pi / 2}\right\rangle+i \frac{\pi \epsilon}{2 \Omega}\left\langle y_{0}\right\rangle,
$$

i.e., the difference between the numbers of atoms in the two wells determines the quadrature phase of one of the matter fields. This method is, however, sensitive to the exact determination of the reference phase $\theta$, which for condensates is a central problem 16. Here it is simply assumed that the reference phase can be determined by the experimentalist. With such a quadrature matter field 
measurement at hand it is possible to distinguish between coherent states like $|\alpha\rangle$ and $|-\alpha\rangle$, which is the necessary resource to apply to both target and mode 2 and thus distinguish between the many states of the superposition of Eq.(11). Remark that the requirement for a small number of particles $(N \ll \Omega / \kappa)$ avoids the regime of self-trapping, as observed in [11], when the approximate solution, Eq. (16), is no longer valid. This requirement imposes a severe limitation of this detection method to the "size" of the BEC to be teleported.

\section{RECEIVER OPERATIONS}

The two bits of classical information obtained as described above are transferred to the receiver mode 3 . Now one has to apply the operations needed to transform the condensate state mode 3 in the receiver station into the required state. Depending on the results of the joint homodyne measurement described above, the condensate mode 3 is left in one of the following states,

$$
\begin{array}{r}
\int d^{2} \beta b_{\beta}(A|\beta\rangle+B|-\beta\rangle) \\
\int d^{2} \beta b_{\beta}(A|\beta\rangle-B|-\beta\rangle) \\
\int d^{2} \beta b_{\beta}(A|-\beta\rangle+B|\beta\rangle) \\
-\int d^{2} \beta b_{\beta}(A|-\beta\rangle-B|\beta\rangle)
\end{array}
$$

In order to transform the condensate states (21), 22, and (23) into the required state (20), operations of parity and displacement 13], in principle, can be realised, as described below.

\section{A. Parity}

A parity operation involving only atomic systems may be envisaged for a two-species BEC with the crosscollision strenght given by $\lambda=U_{A B} / 2 \hbar V_{\text {eff }}$, where $\lambda \neq \kappa=U_{A A} / 2 \hbar V_{\text {eff }}=U_{B B} / 2 \hbar V_{\text {eff }}$ [14]. Supposing the central condensate initially in the state (22) and we require to transform it to $(20)$. The auxiliary condensate is prepared in an arbitrary state expanded also in the coherent basis $|\psi\rangle_{a}=\int d^{2} \xi a_{\xi}|\xi\rangle$. Due to cross-collision the state of this system at time $t=2 \pi / \kappa$ is

$$
\begin{aligned}
|\Phi\rangle_{a c}= & \int d^{2} \beta d^{2} \xi ; b_{\beta} a_{\xi} \sum_{n} \frac{e^{-\frac{1}{2}|\xi|^{2}}}{\sqrt{n !}}\left(\xi e^{-i 2 \pi \frac{\left(E_{0} / \hbar-\kappa\right)}{\kappa}}\right)^{n}|n\rangle_{a} \\
& \otimes\left(A\left|-\beta e^{-i 2 \pi \frac{\left(E_{0} / \hbar-\kappa+n \lambda\right)}{\kappa}}\right\rangle+B\left|\beta e^{-i 2 \pi \frac{\left(E_{0} / \hbar-\kappa+n \lambda\right)}{\kappa}}\right\rangle\right)
\end{aligned}
$$

Now suppose a number projective measurement is made on the auxiliary condensate, projecting it to $|m\rangle$. The normalized conditioned state of the central condensate is

$$
|\Phi\rangle_{c}=\frac{{ }_{a}\langle m \mid \Phi\rangle_{a c}}{\sqrt{\operatorname{Tr}\left\{\left.{ }_{a}\langle m \mid \Phi\rangle_{a c}\right|^{2}\right\}}}
$$

which for $\lambda=\left(E_{0} / \hbar-\kappa\right)=\kappa / 2$ turns out to be

$$
|\Phi\rangle_{c}=\int d^{2} \beta b_{\beta}\left(A\left|\beta e^{i m \pi}\right\rangle+B\left|-\beta e^{i m \pi}\right\rangle\right)_{c},
$$

and now, if $m$ is even the central condensate mode is left in the required state, if it is not the experiment is to be repeated. Thus the number of atoms in the auxiliary condensate mode determines the parity of the central condensate mode. In Fig. 2 we plot the efficiency of this proccess by summing over all the even number probabilities

$$
P_{\text {even }}=\sum_{m=\text { even }} P(m)=\sum_{m=e v e n} \operatorname{Tr}\left\{\left.\left.\right|_{a}\langle m \mid \Phi\rangle_{a c}\right|^{2}\right\},
$$

for the auxiliary mode prepared in three distinct states. The dashed line is for the auxiliary mode prepared in a number state with average number of atoms $\langle n\rangle$. As it is expected in this case the probability of success is constantly 0.5 indepently of the atom number. The dotted line is for a coherent state. We see that when the auxiliary mode is prepared in this state the probability of success also attains the limit of 0.5 , unless the auxiliary mode has less than one atom in average as it attains the vaccum state. However the state that presents the best efficiency is the squeezed vacuum state [6] as depicted by the solid line in Fig. 2, by varying the squeezing parameter $r$ in the top axis of the plot. We see that for $r \leq 1.425$ the probability of success of the event is higher than 0.5. This is probably the best situation for parity control.

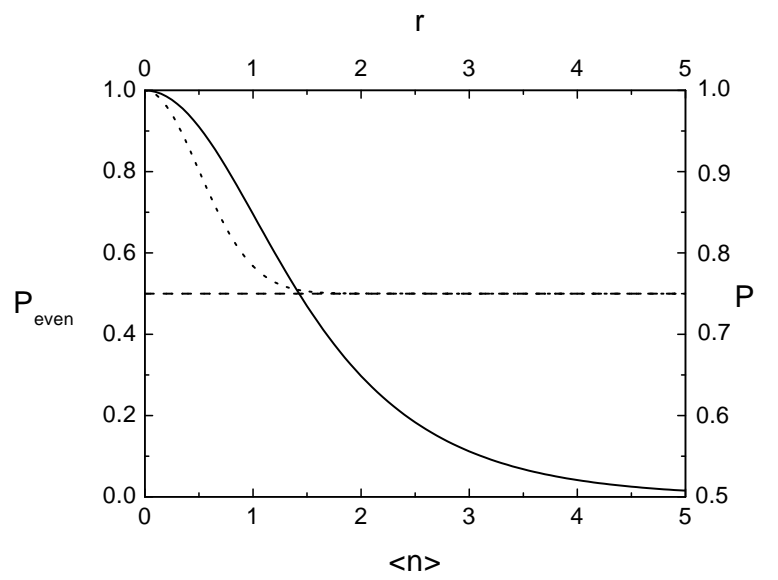

Fig.2. Efficiency of even number count event for the auxiliary mode prepared in number state (dashed line), coherent state (dotted line), and squeezed vacuum state (solid line). Bottom axis represents the number and coherent average number, while the top axis is for the squeezing parameter of the squeezed vacuum. Right axis is for the whole process efficiency for $P_{D}=1$. 


\section{B. Displacement}

Now, to transform the state (21) into (20), we choo a virtual displacement on the central field, defined as $\mathrm{fc}$ lows. Consider an atomic beam acting as a displaceme operator over the central mode

$$
\begin{aligned}
D_{\delta}|\Phi\rangle_{c}= & \int d^{2} \beta b_{\beta}\left\{\cos \left[\operatorname{Im}\left(\delta^{*} \beta\right)\right](A|\beta+\delta\rangle-B \mid-\beta+\right. \\
& +i \sin [\operatorname{Im}(\delta \beta)](A|\beta+\delta\rangle+B|-\beta+\delta\rangle)\} .
\end{aligned}
$$

Suppose $\delta \in \Re$ and $|\delta| \ll|\beta|$. If $\delta=(l+1 / 2) \pi / \operatorname{Im}(k$ for $l=0,1,2 \ldots$, the central mode state is left in $\mathrm{t}$. required state (but a global phase of no importance The displacement operation can also be directly given 1 quadrature $\left(X=b+b^{\dagger}\right)$ measurement through the hom dyne detection described in Sec. IV. As the paramet $|\delta| \ll|\beta|$ is known to be very small, the displaceme operator, is given by

$$
D_{\delta}=e^{i|\delta| X} \approx 1+i \delta X ;\left[D_{\delta}, X\right]=0 .
$$

Knowing $\delta$, the measurement of $X$ gives the required $\mathrm{d}$ : placement. The efficiency of this proccess is depende upon the full knowledge of the above constants, and th upon experimental mastering.

Rotations such as that proposed above can be realis by virtual displacement. Obviously, the state (23) ci be transformed into (20) by sequential applications the displacement and parity operations. With this pr cedure, the teleportation protocol is complete. Noti however that none of the above operations are unitar being dependent on selective measurements, and thus reversible. A figure of merit of the whole proccess can 1 given by adding the probability of succes of each event and dividing by the number of equiprobable events,

$$
P=\left(1+P_{\text {even }}+P_{D}+P_{\text {even }} P_{D}\right) / 4,
$$

where $P_{D}$, the probability of success of the displacement operation, is determined by the experimental control. In the right axis of Fig. 2 we compare $P$ for the three states considered above for the auxiliary mode, fixing $P_{D}=1$. Again we observe that for $r \leq 1.425$ the squeezed vacuum is the best state for the auxiliary mode to be prepared. In Fig. 3 we analyse $P$ for the squeezed vacuum by varying both $r$ and $P_{D}$. The efficiency decreases considerably (less than 0.3) when both, the squeezing parameter is high and the displacement process efficiency is low. But, when the squeezing parameter is fixed to 0 , or alternatively $P_{D}$ is fixed to 1 , either situations have efficiency higher than 0.5 attaining the ideality for $P_{D}=1$ and $r=0$, the vacuum state. This situation corresponds to an empty mode. Thus if the auxiliary mode can be initially prepared in a vacuum state the protocol has high probability of success.

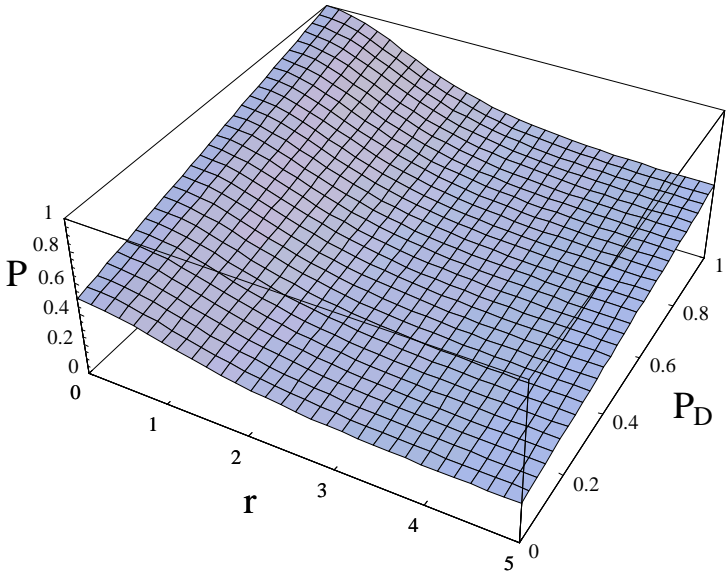

Fig.3. Efficiency of success of operation for the auxiliary mode prepared in a squeezed vacuum state.

\section{PHYSICAL IMPLEMENTATION}

A specific physical implementation of the time dependent potential can be designed, similarly to what is presented in [17] on optical lattices. Let us consider a picture of an atom driven on a $|J=1 / 2\rangle \rightarrow|J=3 / 2\rangle$ transition by a one-dimensional optical lattice red-detuned in 1D lin-angle-lin configuration. The optical field can be written as a superposition of opposite helicity standing waves 17,18

$$
\begin{aligned}
\mathbf{E}_{L}(z)= & \sqrt{2} E_{1}\left[-e^{-i \theta / 2} \cos \left(k_{L} z+\theta / 2\right) \mathbf{e}_{+}\right. \\
& \left.+e^{i \theta / 2} \cos \left(k_{L} z-\theta / 2\right) \mathbf{e}_{-}\right],
\end{aligned}
$$

for a convenient choice of relative phase between the beams. The potential for atoms in the ground state is

$$
\begin{aligned}
U(z)= & -\frac{2 U_{1}}{3}\left\{2\left[1+\cos \theta \cos \left(2 k_{L} z\right)\right] I\right. \\
& \left.+\left[\sin \theta \sin \left(2 k_{L} z\right)\right] \sigma_{z}\right\}-\frac{\hbar}{2} \gamma \mathbf{B} \cdot \sigma,
\end{aligned}
$$

where $U_{1}$ is the light shift produced by a single beam of amplitude $E_{1}$, while $\mathbf{B}$ is a magnetic field and $\left\{I, \sigma_{i}\right\}$ are the identity and Pauli spin operators in the ground-state manifold. By varying $\theta$ the peak-peak modulation depth of the potential and the distance between the $|m=1 / 2\rangle$ and $|m=-1 / 2\rangle$ potential wells are changed by

$$
U_{p}=\frac{4}{3} U_{1} \sqrt{3 \cos ^{2} \theta+1}, \quad k_{l} \Delta z=\tan ^{-1}\left(\frac{\tan \theta}{2}\right),
$$

respectively, while changing the longitudinal component of $\mathbf{B}$ shifts the minima of these wells. The transverse component of $\mathbf{B}$ breaks the degeneracy of the bipotential 
at positions of linearly polarized light. By varying $\theta$ appropriately one can design adiabatically time dependent potentials [12,17]. By loading the optical potential with the condensate at $\theta=\pi / 2$, and changing it from $\pi / 2$ to $5 \pi / 2$ adiabatically, we obtain the sequential controlled collision we required, as pictured in Fig. 4. The three condensate modes should be loaded in neighbouring wells in order to produce the required protocol.

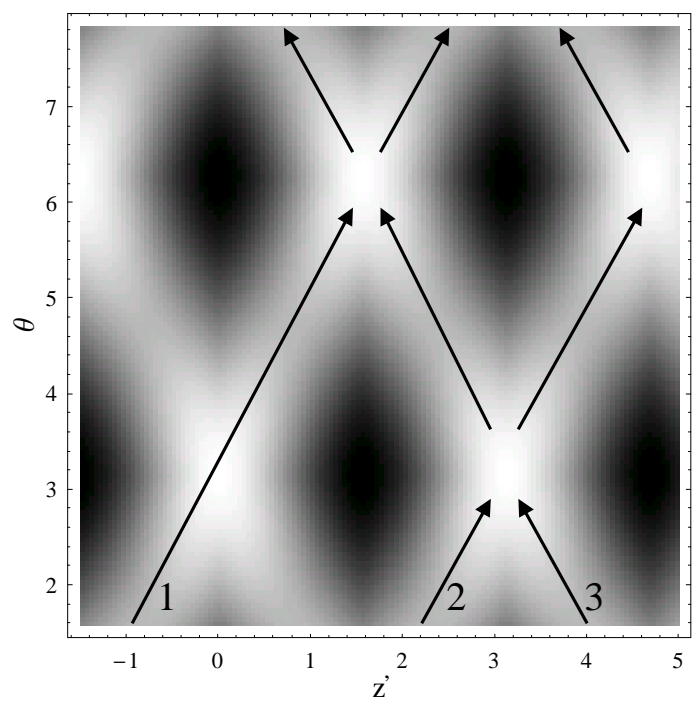

Fig.4. Density map of the $m=1 / 2$ optical lattice trap potential. $B_{\|}=0$, while $B_{\perp} \neq 0$. Brighter zones corresponds to potential minima, $z^{\prime}=2 k_{L} z$. The sequential collision path is depicted by the numbered arrows.

An alternative approach can be designed much in the same way of the controlled entanglement generation in atomic ensembles of Jaksch et al. 12, with no need of a magnetic field, but where different internal spin components are selected to promote the minima of the potential to move in relation to each other. Adiabaticity here, is then related to the speed of the the minima displacement. This alternative has an advantage in relation to the first one, which is that the condensate modes do not need to be loaded in neighbouring wells, as longer as the condensate modes 1 and 3 have the same spin component, the mode 2 with an opposite component, can be moved in relation to the other two modes effecting the required operations, despite their (mode 1 and 3 ) relative position.

\section{DISCUSSION}

In summary, we have speculated about teleportation of a BEC state between modes in a three-well potential (over short distances). Hard-sphere cross-collision is used as a resource to generate entanglement between modes. For the final state measurement, the protocol employs a homodyne detection of the BEC quadrature, in which Josephson coupling of the condensate mode to an auxiliary mode in a neighbouring well plays the role of atomic beam-splitter.

The teleportation protocol itself was constituted of three stages, (i) entanglement formation; (ii) measurement; and (iii) receiver operations. In (i) we limited ourselves to the situation of adiabatic time varying potential $\left(\frac{d V}{d t} \ll\left(E^{\prime}-E_{0}\right) / \hbar\right)$ to avoid excitations to other states. In (ii) the homodyne detection of the BEC quadrature guarantees that for $(N \ll \Omega / \kappa)$, a well-diluted atomic gas and short time of interaction, a condensate mode quadrature can be determined by the difference of atoms between the central mode and an auxiliary mode. In (iii) parity and displacement operations were proposed to fix the teleported state at the receiver station. Since those operations are dependent upon selective measurements, the whole teleportation protocol is limited by the operations efficiencies. The efficiency of the parity operation is given by the even-parity of number of atoms present in the auxiliary state, which can be higher than 0.5 once the auxiliary condensate can be prepared approximately to a vacuum squeezed state with squeezing parameter $r \leq 1.425$. Together with the virtual displacement operations, those parity operations have their efficiency limited by the full knowledge of the experimental parameters. Such is the case for the squeezing parameter, $r$, and the virtual displacement $\delta=(l+1 / 2) \pi / \operatorname{Im}(\beta)$, for $l=0,1,2 \ldots$. The efficiency of the whole operation vary from 0.3 to 1 for auxiliary mode prepared in the squeezed vacuum, by varying $r$ and the probability of success of the displacement operation.

We stress that the proposed protocol is idealised in that the above measurement processes will be very sensitive to the presence of dissipation, such as collisions with non-condensate atoms [19]. We expect that, together with the requirement of a small number of particles at the measurement stage $(N \ll \Omega / \kappa)$, decoherence effects impose a severe limitation to the "size" of the BEC to be teleported. The competition between Josephson coupling and cross-collision could also represent a limitation for the above protocol. We know that if the modes are prepared in coherent states this does not represent any problem, once the Josephson coupling just adds a phase to the state. However for other states this may not be so. Thus the competition of those evolutions need to be further investigated.

As a last remark, throughout the paper we have assumed a coherent basis representation for the condensate mode states, which are better adapted to our discussion. Although not specifically adressing to coherent states for the modes, those states could also be considered, if each mode were actually entangled with another condensate to form an entangled coherent state. The scheme could 
then proceed as described and would effectively be entanglement swapping.

Despite the idealisation, we hope that the above considerations bring some contribution to the realisation of matter field state teleportation in the near future.

\section{ACKNOWLEDGMENTS}

The author thanks G. J. Milburn for encouraging comments, and M. H. Y. Moussa and G. A. Prataviera for enlightening discussions. This work is supported by FAPESP-Brazil, under projects 01/00530-2 and 00/15084-5.

[1] C.H. Bennett, G. Brassard, C. Crepeau, R. Jozsa, A. Peres, and W.K. Wootters, Phys. Rev. Lett. 70, 1895 (1993).

[2] D. Bouwmeester J.-W. Pan, K. Mattle, M. Eibl, H. Weinfurter, and A. Zeilinger, Nature(London) 390, 575 (1997).

[3] P. G. Kwiat, K. Mattle, H. Weinfurter, A. Zeilinger, A. V. Sergienko, and Y. Shih, Phys. Rev. Lett. 75, 4337 (1995).

[4] E. Hagley, X. Maître, G. Nogues, C. Wunderlich, M. Brune, J. M. Raimond, and S. Haroche, Phys. Rev. Lett. 79, 1 (1997); A. Rauschenbeutel, G. Nogues, S. Osnaghi, P. Bertet, M. Brune, J.M. Raimond, and S. Haroche, Science 288, 2024 (2000); C.A. Sackett, D. Kielpinski, B.E. King, C. Langer, V. Meyer, C.J. Myatt, M. Rowe, Q.A. Turchette, W.M. Itano, D.J. Wineland, and C. Monroe,
Nature 404, 256 (2000); B. Julsgaard, A. Koxhekin, and E.S. Polzik, Nature 413, 400 (2001).

[5] A. Kuzmich and E.S. Polzik, Phys. Rev. Lett. 85, 5639 (2000); Lu-Ming Duan, J. I. Cirac, P. Zoller, and E. S. Polzik, ibid.85, 5643 (2000).

[6] A.S. Parkins and D.F. Walls, Phys. Rep. 303, 1 (1998).

[7] J. Javanainen, Phys. Rev. Lett. 57, 3164 (1986); A. Smerzi, S. Fantoni, S. Giovanazzi, and S. R. Shenoy, ibid. 79, 4950 (1997).

[8] M.H. Anderson, J.R. Ensher, M.R. Matthews, C.E. Wieman, and E.A. Cornell, Science 269, 198 (1995); K.B. Davis et al., Phys. Rev. Lett. 75, 3969 (1995).

[9] M. Barrett, J. Sauer, and M.S. Chapman, Phys. Rev. Lett. 87, 010404 (2001).

[10] W. Hänsel, P. Hommelhoff, T.W. Hänsch, and J. Reichel, Nature 413, 498 (2001).

[11] G.J. Milburn, J. Corney, E.M. Wright, and D.F. Walls, Phys. Rev. A 55, 4318 (1997); J. F. Corney and G.J. Milburn, Phys. Rev. A 58, 2399 (1998).

[12] D. Jaksch et al., Phys. Rev. Lett. 82, 1975 (1999).

[13] M.C. de Oliveira and G.J. Milburn, Phys. Rev. A 65, 032304 (2002).

[14] J.I. Cirac, M. Lewenstein, K. Mølmer, and P. Zoller, Phys. Rev. A 57, 1208 (1998); D. Gordon, and C.M. Savage, ibid. 59, 4623 (1999).

[15] E.L. Bolda, S.M. Tan, and D.F. Walls, Phys. Rev. Lett. 79, 4719 (1997); R. Walser, ibid. 79, 4724 (1997); S. Mancini and P. Tombesi, Europhys. Lett. 40, (1997).

[16] J. A. Dunningham and K. Burnett, Phys. Rev. Lett. 82, 3729 (1999).

[17] I.H. Deutsch and P.S. Jessen, Phys. Rev. A 57, 1972 (1998).

[18] V. Finkelstein, P.R. Berman, and J. Guo, Phys. Rev. A 45, 1829 (1992).

[19] J. Anglin, Phys. Rev. Lett. 79, 6 (1997). 